\title{
A New Passive Repetitive Controller For Discrete-Time Finite-Frequency Positive-Real Systems.
}

\author{
Ramon Costa-Castelló and Danwei Wang and Robert Griñó
}

\begin{abstract}
This work proposes a new repetitive controller for discrete-time finite-frequency positive-real systems which are required to track periodic references or to attenuate periodic disturbances. The main characteristic of the proposed controller is its passivity. This fact implies closed-loop stable behavior when it is used with discrete-time passive plants, but additional conditions must be fulfilled when it is used with a discretetime finite-frequency positive-real plant. These conditions are analyzed and a design procedure is proposed.
\end{abstract}

\section{INTRODUCTION}

The concept of repetitive control has been largely used in different control areas such as CD and disk arm actuators [1], electronic rectifiers [17], pulse-width modulated (PWM) inverters [16], [15], and current harmonics active filters [2].

It is known that the repetitive controller causes a great increase in the order of the system and, then, the closedloop stability is difficult to analyze using algebraic methods. Normally, the stability study of these systems is based on splitting the closed-loop system in three series-connected subsystems which are required to be stable. For two of them, checking the stability is a trivial matter but, for the remaining system the Small Gain Theorem is used to assure its stability [9], [3].

Traditional repetitive controllers are decomposed in two parts: the internal model and the stabilizing controller. Traditional internal model has a big relative degree that implies a great phase lag and a slow response time. This work proposes a new repetitive internal model which is discretetime passive (equivalently, it is Positive Real), so it has relative degree zero. This property implies a limited phase lag. In addition, the proposed internal model does not lose most of the relevant properties in the traditional internal model, so it can be used in a complete or odd-harmonic [6] structure.

As a consequence, it can be used to reject/follow periodic signals in discrete-time passive plants, or plants that can be passivized using feedback [13], without worrying about the overall system closed-loop stability [14]. Additionally, in this work, the use of the proposed repetitive internal model to control a plant which is finite-frequency positive real (FFPR) $[10]$ in the low frequency range is presented.

This work was supported in part by the Comision Interministerial de Ciencia y Tecnología (CICYT) under Projects DPI2004-06871-C02-02.

R. Costa-Castelló and R. Griñó are with the Institut d'Organització i Control de Sistemes industrials (IOC), Universitat Politècnica de Catalunya (UPC), Av. Diagonal 647,11, 08028-Barcelona, SPAIN (email: ramon.costacastello@ieee.org, roberto.grino@upc.edu). D. Wang is with the School of Electrical and Electronics Engineering, Nanyang Technological University (NTU), Singapore 639798 (e-mail: edwwang@ntu.edu.sg).

\section{Proposed Internal Model}

\section{A. The Internal Model}

The proposed internal model is defined by the following transfer function

$$
G(z)=\frac{Y(z)}{U(z)}=k_{r} \frac{z^{N}-H(z) \cdot \beta}{z^{N}-H(z) \cdot \alpha}
$$

where $N \in \mathbb{N}, k_{r} \in \mathbb{R}^{+},-1 \leq \alpha \leq 1,-1 \leq \beta \leq 1$ and $H(z)$ is a low pass filter.

It can be shown that, for $H(z)=1$, the poles of (1) $\operatorname{are}^{1}[4]$ :

$$
p_{k}=\sqrt[N]{|\alpha|} e^{j\left(\frac{2 \cdot \pi}{N} k+\frac{\pi(1-\operatorname{sign}(\alpha))}{2 N}\right)}, k=0,1, \ldots, N-1,
$$

so they are uniformly distributed over a circumference of radius $^{2} \sqrt[N]{\alpha}$. The frequencies associated to the poles are $\omega_{k}=\frac{2 \pi}{N} k+\frac{\pi(1-\operatorname{sign}(\alpha))}{2 N}$, so the poles are placed covering all the harmonic frequencies of the fundamental one, $\frac{2 \pi}{N}$, in the frequency principal period $[-\pi, \pi]$. This pole placement is the same as the one obtained in the traditional internal model. In contrast to the traditional internal model, the proposed one has zeroes, for $H(z)=1$, placed in:

$$
z_{k}=\sqrt[N]{|\beta|} e^{j\left(\frac{2 \cdot \pi}{N} k+\frac{\pi(1-\operatorname{sign}(\beta))}{2 N}\right)}, k=0,1, \ldots, N-1,
$$

so they are also uniformly distributed over a circumference of radius $\sqrt[N]{\beta}$ with associated frequencies $\omega_{k}=\frac{2 \pi}{N} k+$ $\underline{\pi(1-\operatorname{sign}(\beta))}$

The effect of $H(z)$ modifies the poles/zeroes position, trying to leave unchanged the poles/zeros inside the desired passband while reducing as much as possible the module of the poles/zeroes outside the desired band. Although ideally only the poles/zeroes module is designed to be modified, in practice a slightly displacement in the poles/zeroes frequencies is also introduced.

\section{B. The Energetic Structure}

Let $(A, B, C, D)$ be the LTI discrete-time system

$$
\begin{aligned}
x_{k+1} & =A x_{k}+B u_{k} \\
y_{k} & =C x_{k}+D u_{k}
\end{aligned}
$$

where $x_{k} \in \mathbb{R}^{n}, y_{k}, u_{k} \in \mathbb{R}^{m}$; or in input/output form

$$
G(z) \triangleq C\left(z I_{d}-A\right)^{-1} B+D
$$

${ }^{1} \operatorname{sign}(x)$ equals 1 for $x \geq 0$ and -1 for $x<0$.

${ }^{2}|\alpha|<1$ is necessary to assure the stability of the repetitive cell. 
Definition 1 (Discrete Time Passivity (DTP),[12]):

System (4)-(5) is Discrete Time Passive with storage function $V_{k}=\frac{1}{2} x_{k}^{T} P x_{k}$ ( $V$-passive) if, and only if,

$$
\Delta V_{k} \triangleq V_{k+1}-V_{k} \leq y_{k}^{T} u_{k}
$$

Definition 2 ( $(Q, S, R)$ - Dissipative [5]): Dissipative systems with

$$
\Delta V_{k}=y_{k}^{T} Q y_{k}+2 y_{k}^{T} S u_{k}+u_{k}^{T} R u_{k}
$$

with $Q$ and $R$ being symmetric matrices and $S$ an appropriate size matrix, are regarded as $(Q, S, R)$-Dissipative systems.

Lemma 1 ([5]): If a SISO system $(A, B, C, D)$ with the transfer function $G(z)$ is $(Q, S, R)-D T P$ then:

1) If $Q<0$ then the graph of $G\left(e^{j \omega}\right)$ lies inside the circle on the complex plane with center $\frac{S}{|Q|}$ and radius $\left(\frac{1}{|Q|}\right) \sqrt{S^{2}+R|Q|}$.

2) If $Q=0$ then the graph of $G\left(e^{j \omega}\right)$ lies to the right (if $S>0$ ) or the left (if $S<0$ ) of the vertical line $\operatorname{Re}\{z\}=-\frac{R}{2 S}$.

A state-space description, for $H(z)=1$, of the transfer function in equation (1) is

$$
\begin{aligned}
x_{n+1} & =A x_{n}+B u_{n} \\
y_{n} & =C x_{n}+D u_{n}
\end{aligned}
$$

where

$$
\begin{aligned}
& A=\left[\begin{array}{cccccc}
0 & 1 & 0 & 0 & \cdots & 0 \\
0 & 0 & 1 & 0 & \cdots & 0 \\
0 & 0 & 0 & 1 & \cdots & 0 \\
\vdots & \vdots & \vdots & \vdots & \ddots & \vdots \\
0 & 0 & 0 & 0 & \cdots & 1 \\
\alpha & 0 & 0 & 0 & \cdots & 0
\end{array}\right] \\
& B=\left[\begin{array}{llllll}
0 & 0 & 0 & \cdots & 0 & 1
\end{array}\right]^{T} \\
& C=[k(\alpha-\beta), 0,0, \cdots, 0] \\
& D=[k]
\end{aligned}
$$

Proposition 1: The internal model, (1), for $H(z)=1$ for $k_{r}>0,|\alpha| \leq 1,|\beta| \leq 1$ and $\alpha \beta \neq 1$ is $(Q, S, R)-$ Dissipative so DTP.

Proof: Through direct inspection it is possible to see that:

$$
\begin{aligned}
\Delta V_{k} & =\frac{1}{2} x_{k+1}^{T} P x_{k+1}-\frac{1}{2} x_{k}^{T} P x_{k} \\
& =y_{k}^{T} Q y_{k}+2 y_{k}^{T} S u_{k}+u_{k}^{T} R u_{k}
\end{aligned}
$$

with

$$
P=\frac{k_{r}(\beta-\alpha)^{2}}{1-\beta \alpha} \cdot I_{d}
$$

where $I_{d} \in \mathbb{R}^{N \times N}$ stands for the identity matrix and:

$$
\begin{aligned}
Q & =\frac{1-\alpha^{2}}{2 k_{r}(\beta \alpha-1)} \\
R & =\frac{k_{r}\left(1-\beta^{2}\right)}{2(\beta \alpha-1)} \\
S & =\frac{1}{2}
\end{aligned}
$$

Once the values of $Q, S$ and $R$ have been obtained the proof is finished. Note that $Q<0$ and $R<0$ in the interesting cases, so the system is DTP by direct inspection of (8).

Definition 3 (Discrete Time Positive Real (DTPR)): Let $G(z)$ be a square matrix of real rational functions. Then $G(z)$ is called Discrete-Time Positive Real (DTPR) if it fulfills the following properties.

a) The entries of $G(z)$ are analytic in $|z|>1$.

b) Every pole of $G(z)$ on $e^{j \omega}$, if any, is simple and the corresponding residue matrix is Hermitian positive semidefinite.

c) $G\left(e^{j \cdot \omega}\right)+G^{*}\left(e^{j \cdot \omega}\right) \geq 0 \forall|\omega| \leq \pi$.

Remark 1: Is it important to remark that in linear systems DTPR is equivalent to DTP. The connection between both definitions is provided by the discrete-time KYP Lemma [8].

Proposition 2: The internal model, $G(z)=k_{r} \frac{z^{N}-\beta \cdot H(z)}{z^{N}-\alpha \cdot H(z)}$, introduced in equation (1), for $k_{r}>0,|\alpha| \leq 1$, $|\beta| \leq 1, \alpha \beta \neq 1$ with $H(z)$ stable, $H(1)=1$ and $\left|H\left(e^{j \omega}\right)\right|<1$ for $0<|\omega|<\pi$ is DTPR.

Proof: The proof is organized in the following way, first of all it will be proved that the Nyquist plot of $k_{r} \frac{z^{N}-\beta}{z^{N}-\alpha}$ decomposes the complex plane in two connected regions, next it will be proven that the curves $k_{r} \frac{e^{j \cdot \omega \cdot N}-\beta}{e^{j \cdot \omega \cdot N}-\alpha}$ and $k_{r} \frac{e^{j \cdot \omega \cdot N}-\beta H\left(e^{j \cdot \omega}\right)}{e^{j \cdot \omega \cdot N}-\alpha H\left(e^{j \cdot \omega}\right)}$ have only one point in common and, finally, it will be shown that another point is in one of the partitions defined by the Nyquist plot of $k_{r} \frac{z^{N}-\beta}{z^{N}-\alpha}$ such that the curve is PR. This procedure, together with the assumption that the Nyquist plot of $k_{r} \frac{z^{N}-\beta H(z)}{z^{N}-\alpha H(z)}$ is continuous, will prove that $k_{r} \frac{z^{N}-\beta H(z)}{z^{N}-\alpha H(z)}$ is $\mathrm{PR}$.

1) The Nyquist plot of $k_{r} \frac{z^{N}-\beta}{z^{N}-\alpha}$ defines a complex plane partition. In proposition 1 it has been stated that $k_{r} \frac{z^{N}-\beta}{z^{N}-\alpha}$ is DTP, so it is DTPR; i.e. the Nyquist plot of $k_{r} \frac{z^{N}-\beta}{z^{N}-\alpha}$ lies in the open right half complex plane. The concrete topology will be analyzed in two different cases,

- In case $|\alpha|<1$, the Nyquist plot of $G\left(e^{j \cdot \omega}\right)$ is a circumference of radius $r=k_{r} \frac{|\alpha-\beta|}{1-\alpha^{2}}$ and center $c=k_{r} \frac{1-\beta \alpha}{1-\alpha^{2}}$. In this case, the two partitions are the interior and exterior of this circumference.

- In case $|\alpha|=1, \operatorname{Re}\left\{G\left(e^{j \cdot \omega}\right)\right\}=\frac{1+\beta}{2}$. In this case the two partitions are the left and right half plane of the vertical line $z=\frac{1+\beta}{2}$. 
2) The Nyquist plot of $k_{r} \frac{z^{N}-\beta}{z^{N}-\alpha}$ and $k_{r} \frac{z^{N}-\beta \cdot H(z)}{z^{N}-\alpha \cdot H(z)}$ have only one point in common which is the initial point $z=1(\omega=0)$.

To prove this statement lets assume that another intersection exists, so the following equation should be fulfilled:

$$
k_{r} \frac{e^{j \omega_{1} N}-\beta}{e^{j \omega_{1} N}-\alpha}=k_{r} \frac{e^{j \cdot \omega_{2} N}-\beta \cdot H\left(e^{j \cdot \omega_{2}}\right)}{e^{j \cdot \omega_{2} N}-\alpha \cdot H\left(e^{j \cdot \omega_{2}}\right)}
$$

which implies $e^{j \cdot \omega_{2} N}=e^{j \cdot \omega_{1} N} \cdot H\left(e^{j \cdot \omega_{2}}\right)$. The only possible solution is for $\left|H\left(e^{j \cdot \omega_{2}}\right)\right|=1$, according to the hypothesis made on $H(z)$ this is only feasible for $\omega_{2}=0$. Note that if $\alpha=1$ this point is placed at $\infty$.

3) A point of $G(z)$ different from the intersection point is inside one of the partitions.

An interesting point is the last one $z=-1(\omega=\pi)$. This point is $k_{r} \frac{(-1)^{N}-\beta \cdot H(-1)}{(-1)^{N}-\alpha \cdot H(-1)} \in \mathbb{R}$.

- In the case of $|\alpha|<1$ it is sufficient to prove that it is inside the circle described by $k_{r} \frac{e^{j \cdot \omega \cdot N}-\beta}{e^{j \cdot \omega \cdot N}-\alpha}$. In order to fulfill this, it is necessary to fulfill :

$$
\left(\frac{(-1)^{N}-\beta \cdot H(-1)}{(-1)^{N}-\alpha \cdot H(-1)}-\frac{1-\alpha \beta}{1-\alpha^{2}}\right)^{2} \leq \frac{(\alpha-\beta)^{2}}{\left(1-\alpha^{2}\right)^{2}}
$$

which can be rewritten as $-(\alpha-\beta)^{2}(1-$ $\left.H(-1)^{2}\right)<0$ that it is always true for in the proposition conditions $(|H(-1)|<1)$.

- In the case $|\alpha|=1$. It is not difficult to prove that

$$
k_{r} \frac{(-1)^{N}-\beta \cdot H(-1)}{(-1)^{N}-\alpha \cdot H(-1)}-\frac{1+\beta}{2} k_{r}>0
$$

So the Nyquist plot of $k_{r} \frac{z^{N}-\beta \cdot H(z)}{z^{N}-\alpha \cdot H(z)}$ lies in the right half plane of the Nyquist plane.

4) $G(z)$ is analytic in $|z|>1$.

By applying the Small Gain Theorem it can be shown that $G(z)$ stable ( marginally stable for $|\alpha|=1$ ).

It has been proven that the Nyquist plot of $G(z)$ lies in the right half plane of the Nyquist plane and that it is analytic for $|z|>1$ so $G(z)$ is DTPR and, then, DTP.

\section{Frequency Response}

This section details some geometric aspects of the frequency response $\left(G\left(e^{j \cdot \omega}\right)=k_{r} \frac{e^{j \cdot \omega \cdot N}-\beta}{e^{j \cdot \omega \cdot N}-\alpha}, \omega \in[-\pi, \pi]\right)$ of the repetitive cell stated in equation (1). Some of these characteristics can be useful to assess the performance of a closed-loop system by looking its contribution to the shape of open-loop transfer function.

The maximum and minimum gains of the repetitive cell $G(z)$ are $\left|G\left(e^{j \omega}\right)\right|_{\max }=\max \left\{k \frac{1-\beta}{1-\alpha}, k_{r} \frac{1+\beta}{1+\alpha}\right\}$ and $\left|G\left(e^{j \omega}\right)\right|_{\min }=\min \left\{k_{r} \frac{1-\beta}{1-\alpha}, k_{r} \frac{1+\beta}{1+\alpha}\right\}$, respectively. The maximum phase loss of $G(z)$ is $\phi_{\max }=\min _{\omega}\left|\angle G\left(e^{j \omega}\right)\right|=$ $\arctan \left(\frac{(\beta-\alpha) \sqrt{\left(\left(1-\alpha^{2}\right)\left(1-\beta^{2}\right)\right)}}{\left(\alpha^{3}-\alpha\right) \beta-\alpha^{2}+1}, \frac{1-\beta^{2}}{1-\beta \alpha}\right)$ and its is always in the $\left[-\frac{\pi}{2}, \frac{\pi}{2}\right]$ range. The phase minima occur at frequencies
$\omega_{l}=\frac{1}{N} \arccos \left(\frac{\alpha+\beta}{\beta \alpha+1}\right)+\frac{2 \pi}{N} l, l=0,1, \ldots, N-1$ reflected to the $[-\pi, \pi]$ range.

In order to obtain the desired tracking/rejection performance in the closed-loop system the controller must contribute with high gain in the harmonic frequencies, the interesting cases among all the possible values of $\alpha$ and $\beta$ are:

- $0<\alpha<1$ and $-1 \leq \beta<\alpha$

- $-1<\alpha<0$ and $\alpha<\beta \leq 1$

In both cases, the gain offered by the repetitive cell at the harmonic frequencies is greater than one, so it contributes to reduce the closed-loop sensitivity function magnitude at these frequencies.

When $H(z)$ is introduced, the gain is reduced outside the bandwidth of $H(z)$. Differently from the traditional internal model, the gain of the proposed internal model will tend to 1 . Inside the bandwidth of $H(z)$, the proposed internal model frequency response will be similar to one presented (for $H(z)=1$ ), clearly the exact frequency response will depend on the exact $H(z)$.

\section{Proposed Design Procedure}

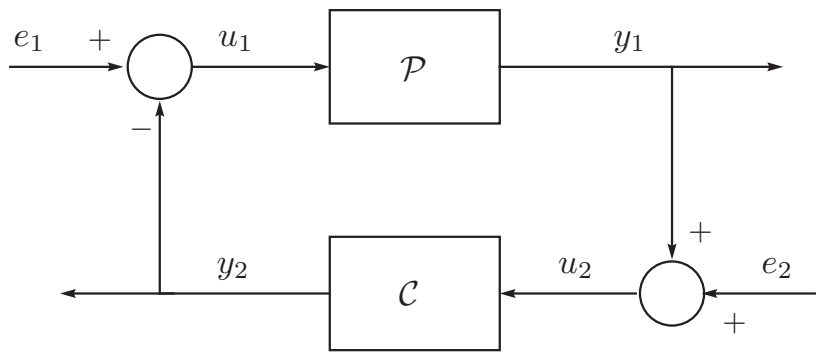

Fig. 1. Feedback connection of two passive systems.

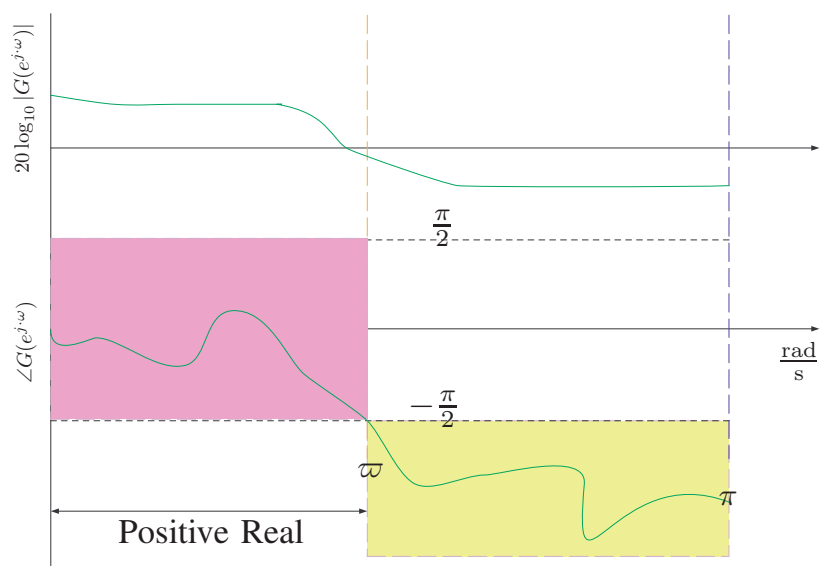

Fig. 2. The concept of finite-frequency positive realness (FFPR).

It is well known that the closed-loop connection of two passive systems generates another passive system [14]. In this 


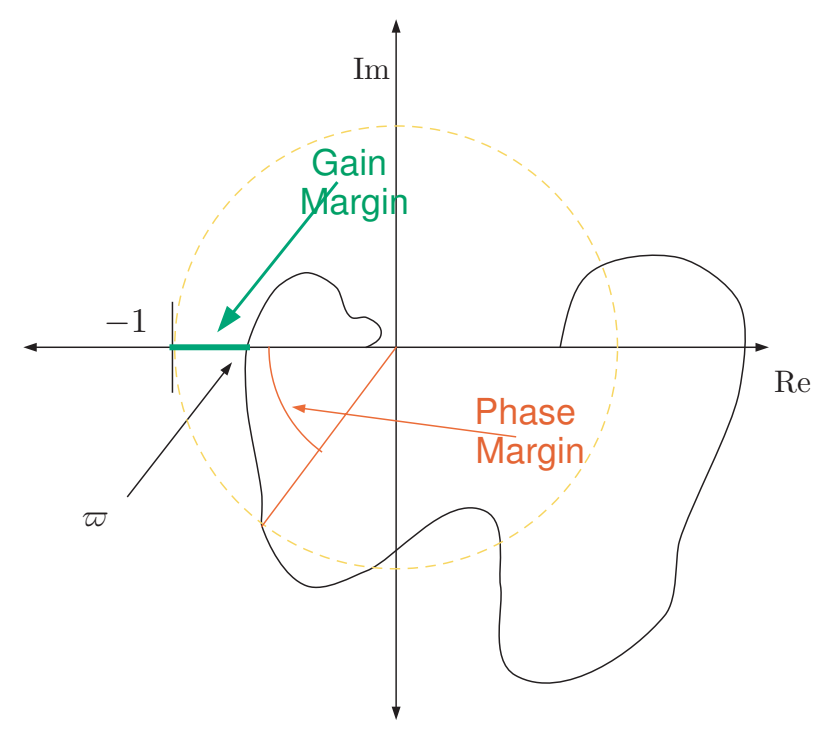

Fig. 3. Worst case open-loop Nyquist plot when connecting to FFPR systems with bandwidth $\varpi$.

sense, the proposed repetitive block $(\mathcal{C})$ can be connected to any discrete time passive $\operatorname{plant}^{3}(\mathcal{P})$, see Fig. 1, giving a discrete time passive closed-loop system. Besides this, the controller structure assures the desired tracking/attenuation performance, for example, any periodic reference applied in $e_{2}$ will be followed by $y_{1}$ with low or zero steady-state error.

Traditionally, repetitive controllers are introduced in a closed-loop system in a plug-in manner [9], [3], i.e. they are used to augment an existing controller. In this kind of connection the repetitive controller is placed in parallel with an unity gain block. Since this system is also passive this property is also preserved if the proposed internal model is used as the repetitive part. So, in Fig. 1 the controller $\mathcal{C}$ could be replaced by the repetitive block in parallel with an unity gain proportional system. Clearly, in this approach, the relation between $\beta$ and the open-loop frequency response will undergo a slight change.

If the plant to be controlled, $\mathcal{P}$, is DTP then the complete stability of scheme is proved by construction. Unfortunately, most discrete time plants are not DTP, especially does which come from a discretized in time continuous-time one (sampled-data system) even when they were passive in continuous time ${ }^{4}$. Unfortunately, conditions needed to passivize a plant imply being relative degree zero [13] so most discrete-time plants cannot be converted into DTP (DTPR) by means of a feedback controller.

In [11] the concept of finite-frequency positive real (FFPR) is introduced.

Definition 4: A transfer function $G(z)$ is called FiniteFrequency Discrete-Time Positive Real (FFDTPR) with

\footnotetext{
${ }^{3}$ Note that $\mathcal{P}$ may be any linear or nonlinear discrete-time passive plant.

${ }^{4}$ Note that DTPR plant has zero relative degree while discretized plants using z-transform and a zoh have relative degree one.
}

bandwidth $\varpi$ if it satisfies conditions a-c in definition 3 with $|\omega|<\pi$ replaced by $|\omega|<\varpi$.

This concept is equivalent to passivity with respect to a certain class of input signals that do not induce fast variations on the states. In [11] it is argued that the FFPR condition it is a necessary condition in order to obtain good closed-loop performance.

It is important to remark that if two FFDTPR systems with bandwidth $\varpi$ are connected in feedback (Fig. 1) the closed-loop system will also be FFDTPR with bandwidth $\varpi$ if it is stable ${ }^{5}$. Unlike the DTPR case, in FFDTPR systems it is necessary to guarantee stability by other means. The stability analysis of this kind of system can be studied in the open-loop Nyquist plot, it is not difficult to see that if two FFDTPR systems with bandwidth $\varpi$ are connected in series, the resultant system will have a phase in the interval $[-\pi, \pi] \mathrm{rad}$, but the negative real axis will not be crossed in the range of frequencies $[0, \varpi]$ (Fig. 3 shows an example of the worst case Nyquist plot when connecting in feedback two FFDTPR systems with bandwidth $\varpi$ ). For frequencies over $\varpi$ the negative real axis may be crossed. In order to guarantee closed-loop stability, it is necessary that if the frequency response curve crosses the negative real axis it will take place to the right of $-1+\mathrm{j} 0$ point, i.e. the small gain theorem is applied in a range of frequencies $[\varpi, \pi]$. This could be written in terms of asking the open-loop function to be bounded real in the complementary range of frequencies where the closed-loop transfer function is asked to be positive real.

Proposition 3: The system obtained when connecting two FFDTPR systems with bandwidth $\varpi, P(z)$ and $C(z)$, in feedback is stable if

$$
\left|P\left(e^{j \cdot \omega}\right)\right|\left|C\left(e^{j \cdot \omega}\right)\right|<1 \quad \forall \omega \in[\varpi, \pi]
$$

Proof: Straightforward from previous comments.

Remark 2: If one of the systems connected in feedback is DTPR, and the other is FFDTPR with bandwidth $\varpi$ the same results apply.

The proposed design procedure is as follows:

1) Given the plant to be controlled, $P(z)$, determine the system bandwidth $(\varpi)$ in which the system is FFDTPR. This bandwidth will determine the closedloop bandwidth. When this bandwidth is not large enough for the desired performance, then $P(z)$ can be combined in series or feedback with a controller which increases the original plant bandwidth. This procedure will define a new $\varpi$.

It is also necessary that the plant (or the modified one ) has very low gain (at least, less than one) in the complementary range of frequencies where it is Positive Real.

2) It is necessary to design a low-pass filter, $H(z)$, such

\footnotetext{
${ }^{5}$ In this cases $\varpi$ is a lower bound in which the positive realness property
} is fulfilled. 
that :

$$
\left|k_{r} \frac{e^{-j \cdot \omega \cdot N}-\beta \cdot H\left(e^{-j \cdot \omega}\right)}{e^{-j \cdot \omega \cdot N}-\alpha \cdot H\left(e^{-j \cdot \omega}\right)}\right|<\gamma \forall \omega \in[\varpi, \pi]
$$

where $\gamma$ is defined in the following way:

$$
\gamma=\inf _{\omega \in[\varpi, \pi]} \frac{1}{P\left(e^{j \cdot \omega}\right)}
$$

in this definition, $P(z)$ includes any series or feedback controller used to improve the original plant characteristics.

\section{NumERICAL EXAMPLE}

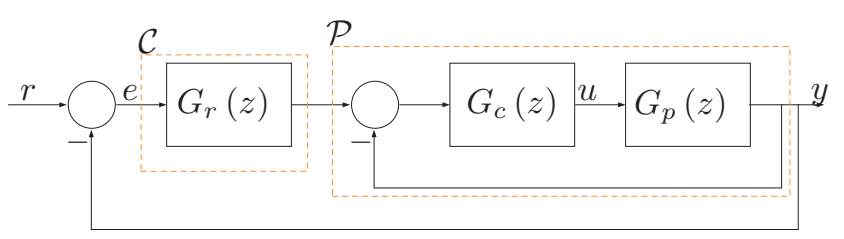

Fig. 4. Complete controller structure.

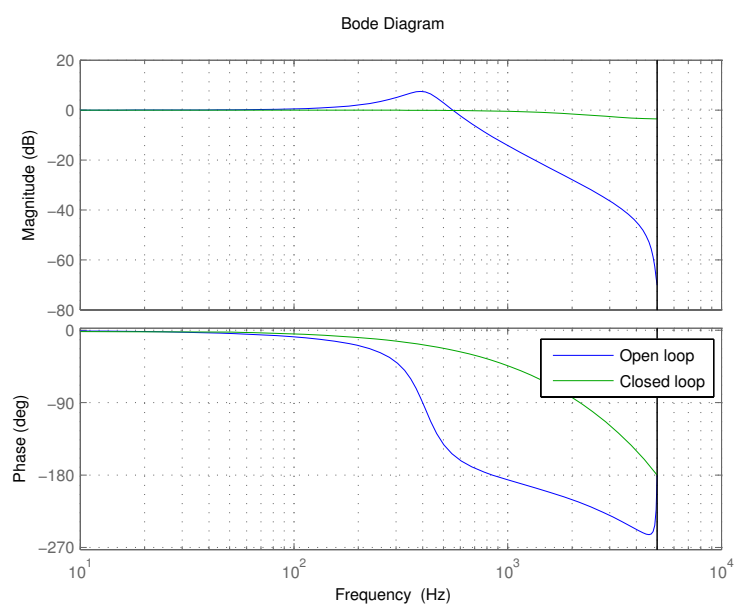

Fig. 5. Open-loop and closed-loop $(\mathcal{P})$ bode plot.

In order to illustrate the proposed procedure a controller for a pulse-width modulated (PWM) DC-AC converter will be developed. Numerical data and models for this example are taken from [16]. The plant under control can be modelled by the following discrete-time transfer function:

$$
G_{p}(z)=\frac{0.03196 z+0.03079}{z^{2}-1.832 z+0.8948}
$$

As it can be seen in Fig. 5 this plant is FFDTPR with bandwith $\varpi=399 \mathrm{~Hz}$ and $\gamma=0.43$. As it would be impossible to design a $H(z)$ filter for this $\gamma$, a feedback controller will be introduced in order to improve this characteristic. A traditional feedback control which has been used for this system is the One Sampling Ahead Preview (OSAP) controller [15] (similar to a deadbeat controller).

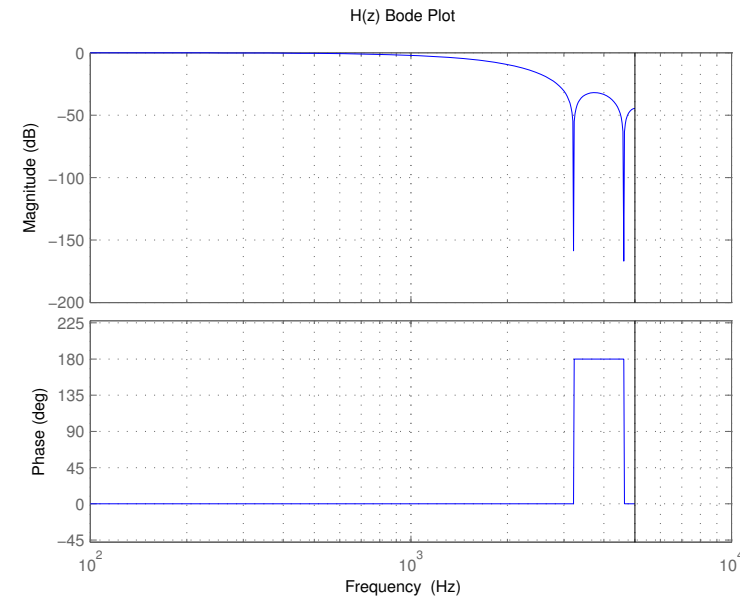

Fig. 6. Open-loop and closed-Loop $(\mathcal{P})$ Bode plot.

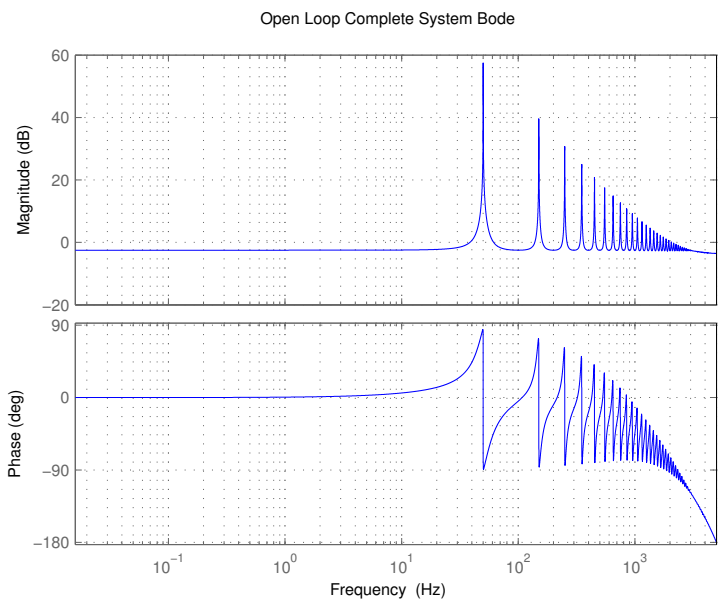

Fig. 7. Complete system open-loop Bode plot.

This controller, $G_{c}$, transforms the complete system in a pure time delay. Although this controller would be interesting for our design, it is more convenient to transform the closed-loop system in a more generic transfer function such as :

$$
G_{o}(z)=\frac{1-\rho}{z-\rho}
$$

for $\rho=0.2$ this plant is FFDTPR with bandwith $\varpi=$ $2.179 \mathrm{kHz}$ and $\gamma=1.22$ (Fig. 5) which clearly are better characteristics than the original ones (other higher order transfer functions can be used if needed). Since now on transfer function $G_{O}$ plays the role of $\mathcal{P}$ (Fig. 4).

As shown in Fig. 4 in this work it placed in series with $\mathcal{P}$. In order to design the repetitive cell, in this work the following parameters will be used: $\alpha=-1$ in order to obtain high performance only in odd-harmonics [6], $\beta=-0.5$ in order to obtain a very selective system and $k_{r}$ will be used to fix a trade-off between robustness and time response [7].

Finally, it is necessary to design the filter, $H(z)$, in a 


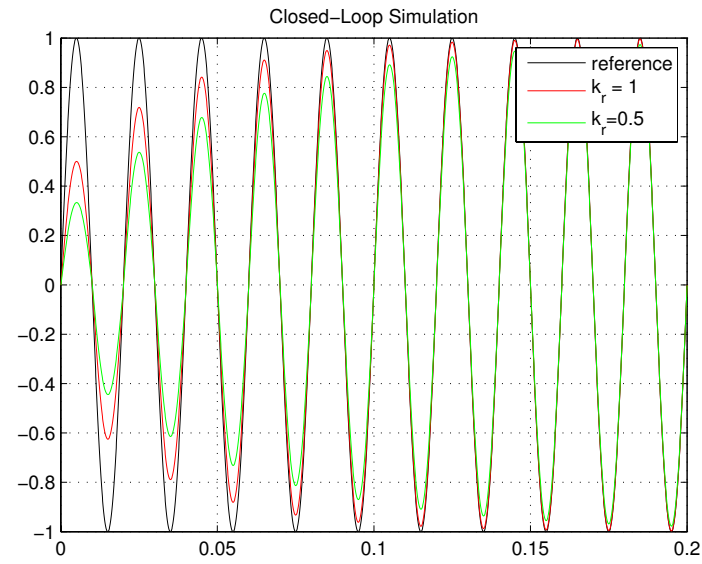

Fig. 8. Closed-loop simulation: output $\left(k_{r}=1\right.$ and $\left.k_{r}=0.5\right)$ and reference vs. time.

proper way following condition 2 in the design procedure. As the internal model (1), must have a maximum gain $\gamma$ it is necessary to obtain a adequate $H(z)$ which make this possible.

When $H(z)$ is equal to a constant $h$ the maximum gain value of the internal model is

$$
\max \left\{k_{r} \frac{1-h \beta}{1-h \alpha}, k_{r} \frac{1+h \beta}{1+h \alpha}\right\}
$$

Making this value equal to $\gamma$, it results possible to obtain:

$$
h=\left|\frac{1-\gamma}{\beta-\gamma \alpha}\right|
$$

So, as a consequence, $h$ stands for the maximum gain the filter, $H(z)$, may introduce in the range of frequencies $\left[\varpi, \frac{\pi}{T s}\right]$. Applying the values of $\gamma, \alpha$ and $\beta$ previously defined results $h=0.73$. To obtain this attenuation a thirdorder null-phase FIR filter has been used (Fig. 6).

In Fig. 7, the open-loop transfer functions is shown. As it can be seen the system has large gain at desired frequencies so good tracking/rejection performances will be obtained at those frequencies.

Finally, the value of $k_{r}$ must be selected. Any value in the interval $[0,1]$ will make the system stable, while value of $k_{r}$ close to 1 will make the system faster and a lower value of $k_{r}$ will improve robustness. Fig. 8 shows the simulation results obtained when a $50 \mathrm{~Hz}$ sinusoidal signal is referenced for a two values of $k_{r}$, as expected in both cases the output track the desired reference.

\section{Conclusions}

A new repetitive internal model has been presented. Its main important characteristic is that it is a Discrete-Time Passive System, i.e. it is Discrete-Time Positive Real. This characteristic implies that the internal model does not introduce the usual huge phase lag of traditional repetitive internal models. Taking advantage of this characteristic the work proposes a new design procedure based on the fact that two FFPR real systems connected in feedback will preserve this property in case the closed-loop systems is stable.

The proposed design procedure allows to include the desired gain margin, while phase margin is assured by the controller and plant structure in the region of interest (where uncertainty is assumed to be low).

Although no constrains are placed on $H(z)$, i.e. any low pass filter can be used. In repetitive control literature, zerophase FIR filters are usually used. A criterion to determine which is the best selection for $H(z)$ in terms of order and system characteristics are currently under study.

\section{ACKNOWLEDGEMENT}

The authors want to thank Prof. Enric Fossas for his comments and helping.

\section{REFERENCES}

[1] K. Chew and M. Tomizuka, "Digital control of repetitive errors in disk drive systems," IEEE Control Systems Magazine, vol. 10, no. 1, pp. 16-20, January 1990.

[2] R. Costa-Castello, R. Griñó, and E. Fossas, "Odd-harmonic digital repetitive control of a single-phase current active filter," IEEE Transactions on Power Electronics, vol. 19, no. 4, pp. 1060-1068, July 2004.

[3] R. Costa-Castello, J. Nebot, and R. Griñó, "Demonstration of the internal model principle by digital repetitive control of an educational laboratory plant," IEEE Transactions on Education, vol. 48, no. 1, pp. 73-80, February 2005.

[4] R. Costa-Castelló and R. Griñó, "A repetitive controller for discretetime passive systems," Automatica, vol. 42, no. 9, September 2006.

[5] G. C. Goodwin and K. S. Sin, Adapative Filtering Prediction and Control, ser. Information and Systems Science Series, T. Kailath, Ed. Englewood Cliffs, New Yersey: Prentice Hall Inc., 1984.

[6] R. Griñó and R. Costa-Castello, "Digital repetitive plug-in controller for odd-harmonic periodic references and disturbances," Automatica, vol. 41, no. 1, pp. 153-157, January 2005.

[7] G. Hillerström and R. Lee, "Trade-offs in repetitive control," University of Cambridge, Tech. Rep. CUED/F-INFENG/TR 294, June 1997.

[8] L. Hitz and B. D. O. Anderson, "Discrete positive-real functions and their applications to system stability," Proc. IEEE, no. 116, pp. 153 $155,1969$.

[9] T. Inoue, M. Nakano, T. Kubo, S. Matsumoto, and H. Baba, "High accuracy control of a proton synchroton magnet power supply," in Proceedings of the 8th IFAC World Congress, 1981, pp. 216-220.

[10] T. Iwasaki and S. Hara, "Generalized kyp lemma: Unified frequency domain inequalities with design applications," IEEE Transactions On Automatic Control, vol. 50, no. 1, pp. 41-59, January 2005.

[11] T. Iwasaki, S. Hara, and H. Yamauchi, "Dynamical system design from a control perspective: Finite frequency positive-realness approach," IEEE Transactions On Automatic Control, vol. 48, no. 8, pp. 13371354, August 2003.

[12] W. Lin, "Synthesis of discrete-time nonlinear systems," Ph.D. dissertation, Washington University, 1993.

[13] Z. Tan, Y. Soh, and L. Xie, "Dissipative control for linear discretetime systems," Automatica, vol. 35, no. 9, pp. 1557-1564, September 1999.

[14] A. van der Schaft, L2-Gain and Passivity Techniques in Nonlinear Control, 2nd ed., ser. Springer Communications and Control Engineering series. Berlin: Springer-Verlag, 2000, vol. 218.

[15] K. Zhou and D. Wang, "Digital repetitive learning controller for three-phase CVCF PWM inverter," IEEE Transactions on Industrial Electronics, vol. 48, no. 4, pp. 820 -830, August 2001.

[16] K. Zhou, D. Wang, and K. S. Low, "Periodic errors elimination in CVCF PWM DC/AC converter systems: repetitive control approach," IEE Proceedings-Control Theory and Applications, vol. 147, no. 6, pp. $694-700$, November 2000.

[17] K. Zhou, D. Wang, and G. Xu, "Repetitive controlled three-phase reversible PWM rectifier," in Proceedings of the 2000 American Control Conference, vol. 1, 2000, pp. $125-129$. 